\title{
3D-Conformal Radiation Therapy and Intensity- Modulated Radiation Therapy Techniques for Laryngeal Cancer Taking Parotid Glands as Organ at Risk
}

\author{
Dler K. Ismael ${ }^{1 *}$, Fatiheea F. Hassan \\ ${ }^{1}$ Department of Basic Sciences, College of Medicine, Hawler Medical University, Erbil, Iraq \\ *Corresponding Author: Dler K. Ismael, M.D., Department of Basic Science- Biophysics Unit, College of Medicine, \\ Hawler Medical University (HMU), Kurdistan, Iraq. Tel: +964-7504813235, Email: i_dler@yahoo.com
}

Received August 17, 2019; Accepted February 17, 2020; Online Published February 29, 2020

\begin{abstract}
Background: Three-dimensional conformal radiotherapy (3D-CRT) and intensity-modulated radiotherapy (IMRT) techniques are used for the treatment of patients with laryngeal cancer.

Objective: This study aimed to investigate the effects of these 2 treatment techniques on the planning target volume (PTV) (laryngeal cancer), dose homogeneity, dose of organs at risk (OARs) (parotid glands), and conformity index.

Methods: This study compared 2 treatment techniques and was conducted from October 2018 to April 2019 at the Zhianawa Cancer Center (ZCC), Sulaimaniyah, Iraq. Eight patients with laryngeal cancer were selected for this study. 3D-CRT and IMRT were used to produce the maximum dose of target volume coverage and minimum dose to the parotid glands. Elekta synergy with a photon beam of $6 \mathrm{MV}$ was used for all measurements. Data analysis was performed using the available statistical package of SPSS 25.

Results: The comparison of 3D-CRT with IMRT showed that the mean conformity index value for IMRT was more conformal than the 3D-CRT plan $(0.956,0.945$, respectively), but the homogeneity index was better with 3D-CRT than with IMRT $(0.175,0.202$, respectively). The mean dose for Rt. parotid glands was higher with IMRT than with 3D-CRT (232.71, 23.26, respectively), and in both plans the mean dose was less than 26 Gy (the standard tolerance value). While in the Lt. parotid gland the mean dose was higher with 3D-CRT than with IMRT $(26.95,23.71$, respectively), the mean dose with 3D-CRT was greater than 26 Gy. The amount of PTV was significantly greater with the IMRT technique than with 3D-CRT (52.15 $\pm 1.61,51.09 .4 \pm 0.74$ Gy, respectively).

Conclusion: This study further supports that IMRT has improved the long-term quality of life of patients with laryngeal cancer. Keywords: Radiotherapy, Conformal, Intensity-Modulated, Organs at Risk, Laryngeal Cancer
\end{abstract}

\section{Background}

Radiation therapy is the use of high-energy $\mathrm{x}$-rays or other particles to destroy cancer cells. It can be given both externally and internally. External radiotherapy aims highenergy $\mathrm{x}$-rays at the affected area using a large machine called a linear accelerator; internal radiotherapy involves having radioactive material placed inside the body and is called brachytherapy. ${ }^{1}$

To treat head and neck cancers by radiation, a perfect technique is needed to spare healthy tissue such as the parotid glands. The intensity-modulated radiotherapy (IMRT) technique resolves this problem. Head and neck treatments are the most difficult to plan because of patient anatomy, various targets with different dose prescriptions, and the number of organs at risk (OARs). ${ }^{2}$

Despite its evident limitations when compared with highly modulated techniques, three-dimensional conformal radiotherapy (3D-CRT) is still widely used to treat head and neck cancers. Some more advanced 3D-CRT treatment planning techniques, such as the Bellinzona technique, have been developed to improve dose distribution to planning target volumes (PTVs) and OARs.

IMRT is a high-precision radiotherapy technique that uses a computer-controlled linear accelerator to deliver precise radiation doses to a tumor or to specific areas within a tumor. It allows the radiation dose to conform more precisely to the three-dimensional shape of the tumor by modulating the intensity of the radiation beam in multiple small volumes. ${ }^{3}$ 3D-CRT involves creating 3-D computer images and delivering highly focused radiation to tumors while sparing nearby healthy tissue. ${ }^{4}$

Copyright $($ C 2020 The Author(s). This is an open-access article distributed under the terms of the Creative Commons Attribution License (http:// creativecommons.org/licenses/by/4.0), which permits unrestricted use, distribution, and reproduction in any medium, provided the original work is properly cited. 
The process of radiotherapy starts with scanning the patients, delineating areas of interest, creating treatment plans, and then sending all the data to the machine through a verification system (Mosaiq). An important part of this chain is the plan created in the treatment planning system. Conventional 3D-CRT treatment planning is manually optimized. The treatment planner chooses all beam parameters, such as the number, directions, shapes, and weights of the beams, and the computer calculates the resulting dose distribution.

In the case of IMRT, dose distribution is inversely determined, meaning that the treatment planner has to first decide the dose distribution, and the computer then calculates a group of beam intensities that will produce, as nearly as possible, the desired dose distribution. ${ }^{5}$

The Bellinzona technique (3D-CRT) has been revised to make use of a multileaf collimator (MLC). It consists of 4 main half-beams, and the posterior field can be split into 2 separated half-beams. Of note, the spinal cord cannot be completely shielded due to the constraints of the travel distances of MLC leaves. Originally, a dose of 50 Gy was prescribed to be delivered to the PTV at the International Commission on Radiation Units and Measurements (ICRU) point. The IMRT planning method, unlike the 3D-CRT planning technique, delivers non-uniform beams across the tumor through a sequence of field segments with varying intensities that, in sum, deliver the desired dose distribution. Segments in each field play important roles in treatment with this technique. IMRT can generate a conformal dose distribution and has a steep dose fall-off at the boundary between the tumor and normal structures. ${ }^{6}$

\subsection{Simulation}

Simulation is a process by which the radiation treatment fields are defined, filmed, and marked out on the patient's skin. The simulator is actually a large-bore computed tomography (CT) scanner that is used to contour the body. The images are then sent to the physics department where they arrange the radiation beams and make a customized plan. $^{7}$ All setup information is documented to complete the patient's treatment record. This is an integral part of the planning process.

\subsection{Qualitative Plan Evaluation}

Quality assurance in radiotherapy includes all procedures that ensure consistency of the medical prescription and safe fulfillment of the prescription as regards the dose to the target volume together with minimal dose to normal tissue, minimal exposure to personnel, and adequate patient monitoring aimed at determining the result of the treatment. Quality assurance in radiotherapy is concerned with all aspects of the radiotherapy process and should involve all groups of staff in a cooperative approach, because quality activities are interdependent. ${ }^{8}$

The radiation oncologist must locate and contour the target area as accurately as possible; then, the medical physicist needs to design an optimal plan to deliver the required dose while sparing healthy tissue. Finally, the technologist must make sure that the patient is correctly positioned before treatment begins.

\subsection{Comparative Evaluation of Treatment Plans}

The treatment planning system can create plans with both 3D-CRT and IMRT techniques. The number of beams in 3D-CRT (Bellinzona technique) is more than 4 with $6 \mathrm{MV}$ which is meant to better protect the OARs. The beams are conformed with the help of MLC to the PTV. With the second technique (IMRT), the number of beams is fixed; nine 6-MV photon beams in different angles are used to create the plan. ${ }^{9}$

\section{Objective}

This study aimed to investigate the effect of 3D-CRT and IMRT techniques on PTV (laryngeal cancer), dose homogeneity, dose to OARs (parotid glands), homogeneity index, and conformity index in the target region and in healthy tissue.

\section{Methods}

This study was conducted at the Zhianawa Cancer Center (ZCC), Sulaimaniyah, Iraq, between October 2018 and April 2019 (7 months).

Eight patients with laryngeal cancer were selected for this study, and an ELEKTA linear accelerator (multienergetic) with 80 MLCs was used (Figure 1). 3D-CRT and IMRT techniques were used for all measurements. The OARs (parotid glands) for all patients were contoured by $\mathrm{CT}$ images for the left (Lt) and right (Rt) parotids. Then, treatment plans were generated for each patient using the superposition algorithm of XIO planning system and 6-MV beams from the linear accelerator.

The prescribed dose was 50 Gy to a reference point in the PTV, which could fulfill most of the ICRU 50 recommendations. Note that just one phase of treatment (50 Gy) was calculated.

A reference point was selected in a clinically relevant region of the PTV with a low dose gradient. Other dose points in the PTV were usually added in order to check dose homogeneity. The main planning goals were to keep the dose to the targets as homogeneous as possible and to minimize the dose to the OARs. A set of DVH parameters for each plan was analyzed to evaluate the performance of each technique. ${ }^{10}$

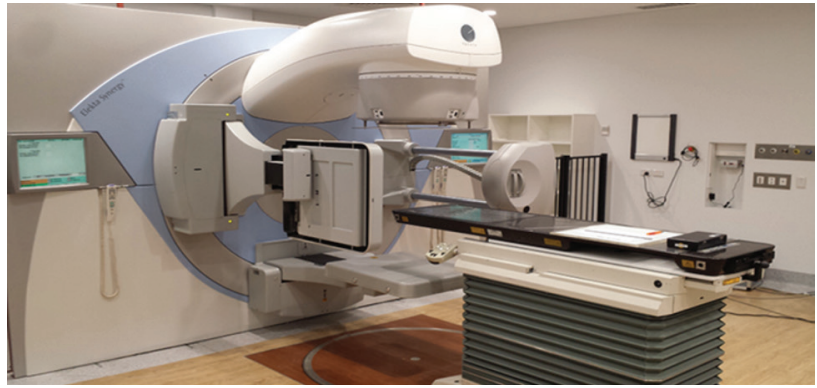

Figure 1. Elekta Synergy Linear Accelerator, ZCC. 


\subsection{General Treatment Strategies}

All patients were treated at the ZCC in Sulaimaniyah, Iraq. Treatment consisted of the following procedures:

1. Assessment before radiotherapy,

2. Diagnosis of tumor type, size, and staging,

3. Determination of OARs (parotid glands),

4. C.T. simulation, contouring, prescription of dose, and fractioning,

5. Planning process,

6. QA for procedure delivery,

7. Delivery,

8. Follow up and assessment of patient during treatment,

9. Regular post-treatment patient follow up for a specified time,

10. Determining short- and long-term side effects/ benefits of radiation exposure to each patient.

In 3D-CRT, the tumor and all organs are viewed in three dimensions. The radiation is delivered by using irregular beams with uniform intensity according to the tumor shape from different directions, and the irradiated volume is conformed to the tumor shape (Figure 2). This conformity increases the ability to deliver higher doses safely to the tumor and minimizes the radiation exposure to the surrounding healthy tissue. ${ }^{11}$

\subsection{Two Types of Treatment Planning}

The first technique used on the eight patients in this study was 3D-CRT, or the Bellinzona technique. This technique is dependent upon the Gantry and collimator angles to apply radiation beams on the parotid glands and is generally started by 4 main diagonal fields (Figure 3 ):

1. Gantry angle of 40 degrees and collimator angle of 90 degrees (first beam, B1);

2. Gantry angle of 135 degrees and collimator angle of 270 degrees (second beam, B2);

3. Gantry angle of 220 degrees and collimator angle of 90 degrees (third beam, B3);

4. Gantry angle of 310 degrees and collimator angle of 270 degrees (fourth beam, B4).

The long-term effects of radiation depend on the irradiation technique, dose, and location. The squamous cells of head and neck tumors usually spread in the neck area (loco-regional); thus, treatment needs to be performed on both sides of the neck. ${ }^{13}$

Elekta's $\mathrm{XiO}$ has a great potential to provide a strong planning system for radiotherapy treatment with the 3D-CRT technique. $\mathrm{XiO}$ is good for precision planning and fluent workflows and can satisfy the expectations of Elekta treatment planning, such as easy integration, advanced dose calculations, high degree of flexibility, automation tools, etc. It also has a variety of workflow tools for planning with contouring, virtual, fusion, and review capabilities. ${ }^{14}$

For PTV, the mean doses of D95\%, D98\%, and D2\% were taken into account, whereas, for OARs, the maximum point dose and the mean dose to the left and right parotid glands were considered.

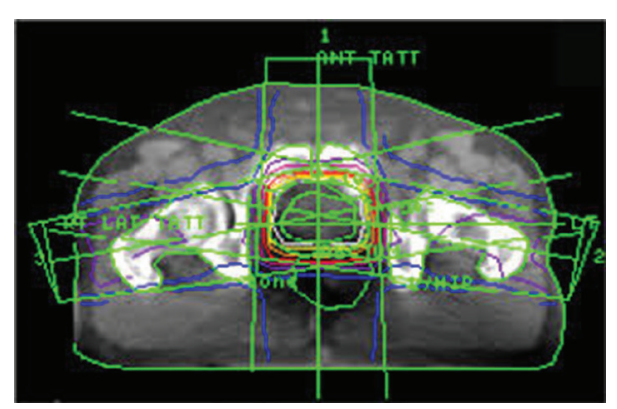

Figure 2. 3D-CRT Plan Using Multiple Beams From Different Directions. ${ }^{11}$

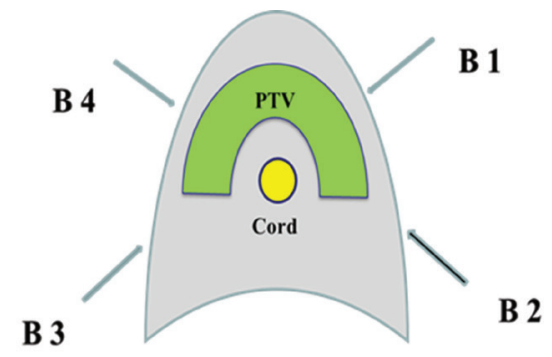

Figure 3. Schematic Illustration of Bellinzona Technique. ${ }^{12}$

The plans with both technique treatments were done in one phase. The plan is a simple box technique with four 6-MV beams that are conformed with the help of MLC to the PTV. ${ }^{15}$

IMRT was the second technique used on the eight patients in this study. It was also done in one phase, i.e. there were no additional doses of radiation. Nine 6-MV coplanar beams with angles of $0^{\circ}, 40^{\circ}, 80^{\circ}, 120^{\circ}, 160^{\circ}$, $200^{\circ}, 240^{\circ}, 280^{\circ}$, and $320^{\circ}$ were used to create the plan. Nine coplanar beams were used for IMRT plans. More small beams (segments) created all the beams in order to modulate the intensity so as to achieve the desired dose distribution. When the plans were finished, those of both methods were compared first according to dose-volume histogram and then according to the time consumed for quality control procedures. Much less time was needed to treat patients using the 3DCRT technique than with the IMRT technique. ${ }^{16}$

The dose constraints were programed according to the ALARA (as low as reasonably achievable) principle for the OARs. The Monte Carlo dose calculation algorithm was used to calculate the dose with a minimum segment area of $4 \mathrm{~cm}^{2}$ and grade space of $3 \mathrm{~mm}$.

With IMRT, Monaco planning was used; Monoco is a treatment planning system (TPS) software for radiotherapy produced by Elekta. It calculates the IMRT plan with high accuracy using the Monte Carlo algorithm (the most accurate dose calculation available). Additionally, it can generate QA plans from an original blueprint for checking the quality of an IMRT plan. Monaco also performs segment shape optimization for smoothing and clustering segments, which can enhance beam weights and shapes, thereby improving plan quality. ${ }^{17}$ 
Monaco TPS version 5.00.02 is used at the ZCC. It works on a network of three main high-performance computers (Quad-Core Intel Xeon 2.93GHz processor, 24GB DDR3 RAM, 4TB Storage) and is connected to the center's main network.

With the IMRT treatment technique, the collimation leaves (MLCs) are carefully adjusted according to the shape, size, and location of the tumor, just like with 3D-CRT, but the intensity of each beam is modulated (not uniform) during treatment. ${ }^{18}$

Several radiation beams are used in IMRT (Figure 4) to focus a higher dose of radiation on the tumor but a much smaller dose on the surrounding normal tissues, reducing adverse events as compared to standard conformal radiotherapy.

The treatment systems use the MLCs not only to shape the radiotherapy area so that it conforms precisely to the form of the tumor, but also to modulate the intensity of the radiation beam during each treatment. ${ }^{19}$

\subsection{Statistical Analysis}

Data was analyzed using the available statistical package SPSS-25 (Statistical Packages for Social Sciences, version 25). Data is presented in simple measures of frequency, percentage, mean, standard deviation, and range (minimum-maximum values).

The variation significance of different means (quantitative data) was tested using the paired t-test for the difference of paired observations (or 2 dependent means). Statistical significance was considered whenever the $P$ value was equal to or less than 0.05 .

\section{Results}

\subsection{Demographic Data}

Eight patients with laryngeal cancer were included in this study. The mean PTVs of all patients were 51.09 Gy $( \pm 0.74)$ and $52.15 \mathrm{~Gy}( \pm 1.61)$ with the $3 \mathrm{D}$-CRT and IMRT techniques, respectively. All information about each patient, e.g., cancer type and stage, was taken into account by radiation oncologists when prescribing doses. The role of medical physicists is to implement ideal planning to distribute the dose prescribed for the target area and reduce the dose received by healthy tissue.

\subsection{Conformity Index}

Conformity index (CI) is defined as the ratio between the volumes covered by a certain dose to the PTV volume. In this study, the volumes covered by $95 \%$ of the prescribed doses were used to calculate CI values (Eq 1). The CI value indicates the degree of conformity of the plan. If CI $<1$, the PTV is under coverage; if $\mathrm{CI}>1$, the normal tissues are receiving a high dose; and if $\mathrm{CI}=1$, the prescribed dose is conformal with the shape of the PTV. ${ }^{20}$

$$
C I=\frac{\text { Volume covered by } 95 \% \text { prescribed dose }}{\text { Volume of PTV }}
$$

\subsection{Homogeneity index}

The homogeneity index (HI) is an objective tool used

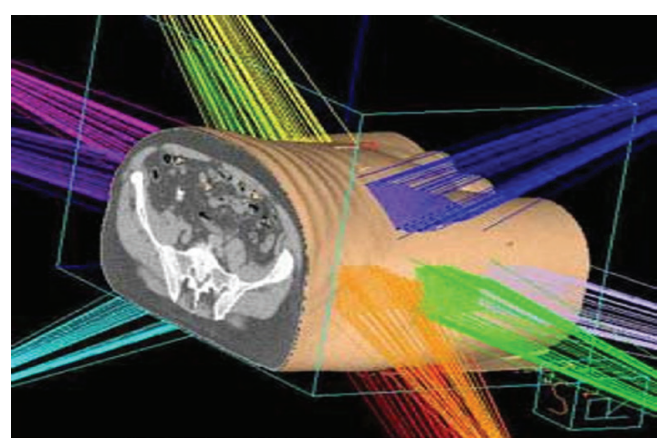

Figure 4. Multiple Beams of Radiation Used for IMRT plan. ${ }^{19}$

to analyze the uniformity of dose distribution in target volumes. The values of $\mathrm{D}_{2 \%}$ and $\mathrm{D}_{98 \%}$ for PTVs were obtained from DVH. $\mathrm{D}_{2 \%}$ represents the maximum dose that will be delivered to $2 \%$ of the PTV, $\mathrm{D}_{\mathrm{p}}$ is the prescribed dose for the PTV, and $\mathrm{D}_{98 \%}$ is the minimum dose calculated for $98 \%$ of the PTV.

These parameters were used to calculate the HI using Eq. 2.

$H I=\frac{D_{2 \%}-D_{98 \%}}{D_{p}}$

A lower HI indicates a better and more uniform dose distribution can be achieved in the target. ${ }^{21}$

As shown in Table 1, dose homogeneity was measured by HI. The results indicated a more homogeneous dose distribution in PTV for patients treated with IMRT than those treated with 3D-CRT (the lower the HI, the optimal the dose homogeneity). Table 1 also shows that HI was slightly improved with IMRT compared with 3DCRT (0.175 vs. 0.2025$)$.

A lower $\mathrm{HI}$ means that a better and more uniform dose distribution can be achieved in the target. ${ }^{22}$

Data from Table 1 indicates that CI was slightly improved with IMRT compared with 3D-CRT (0.956 vs. 0.945$)$. It further indicates that the CI of patient number 8 equaled 1 , which means the optimum conformation in this case.

Also seen in Table 1, the received mean dose volume of the Rt. parotid by IMRT was higher than that by 3D-CRT $(23.71 \pm 3.34,23.26 \pm 11.78$ Gy, respectively), and with both techniques, the mean dose (Rt. parotid) was $<26$ Gy (tolerance mean dose).

Table 1 also shows that the received mean dose volume of the Lt. parotid was higher by $3 \mathrm{D}$-CRT than by IMRT $(26.95 \pm 7.18,23 \pm 1.59 \mathrm{~Gy}$, respectively), and with the 3D-CRT technique, the mean dose to the Lt. parotid was $>26 \mathrm{~Gy}$ (tolerance mean dose). With the IMRT technique, however, the mean dose to the Lt. parotid was $<26 \mathrm{~Gy}$ (tolerance mean dose).

In Table 2, the HI for both techniques are compared. The $P$ values are equal, but the HI for the IMRT technique $(0.19)$ was better and more homogenous than that for the 3D-CRT technique (0.20).

As shown in Table 3 above, the mean values of the Rt. parotids were 23.54 Gy and 24.11 Gy with the 3D-CRT technique and IMRT technique, respectively. Clearly, 
Table 1. Mean and SD (Range) for the Values of the Conformity Index, Homogeneity Index, Mean Dose of Lt and Rt Parotids in 3D-CRT and IMRT Techniques for All 8 Patients

\begin{tabular}{|c|c|c|c|c|c|c|c|c|}
\hline \multirow{2}{*}{$\begin{array}{l}\text { HI } \\
\text { 3DCRT }\end{array}$} & \multicolumn{3}{|c|}{$\mathrm{Cl}$} & \multicolumn{2}{|c|}{ Lt. Parotid Mean Dose (Gy) } & \multicolumn{2}{|c|}{ Rt. Parotid Mean Dose (Gy) } & \multirow[t]{2}{*}{ Tolerance Dose Value of Parotid (Gy) } \\
\hline & IMRT & 3DCRT & IMRT & 3DCRT & IMRT & 3DCRT & IMRT & \\
\hline 0.30 & 0.22 & 0.95 & 0.98 & 40.73 & 26.08 & 36.51 & 26.78 & 26 \\
\hline 0.18 & 0.18 & 0.95 & 0.96 & 28.40 & 24.97 & 32.22 & 16.29 & 26 \\
\hline 0.17 & 0.15 & 0.95 & 0.98 & 31.29 & 23.77 & 29.02 & 23.73 & 26 \\
\hline 0.23 & 0.22 & 0.95 & 0.81 & 29.06 & 21.87 & 32.43 & 24.95 & 26 \\
\hline 0.15 & 0.13 & 0.95 & 0.96 & 23.34 & 23.08 & 1.04 & 26.59 & 26 \\
\hline 0.17 & 0.16 & 0.95 & 0.99 & 24.75 & 23.74 & 15.81 & 25.04 & 26 \\
\hline 0.25 & 0.24 & 0.93 & 0.97 & 17.72 & 21.37 & 22.84 & 24.03 & 26 \\
\hline 0.17 & 0.10 & 0.93 & 1 & 20.36 & 24.86 & 16.23 & 22.32 & 26 \\
\hline 0.2025 & 0.175 & 0.945 & 0.956 & 26.95 & 23.71 & 23.26 & 23.71 & 26 \\
\hline 0.05203 & 0.048 & 0.009 & 0.060 & 7.18 & 1.59 & 11.78 & 3.34 & \\
\hline
\end{tabular}

Table 2. Mean value and $P$ Value of Homogeneity $(\mathrm{HI})$ in 3D-CRT and IMRT Techniuqes

\begin{tabular}{|c|c|c|c|c|c|c|}
\hline & \multicolumn{6}{|c|}{ Test Value $=0$} \\
\hline & \multirow{2}{*}{$\mathbf{T}$} & \multirow{2}{*}{$d f$} & \multirow{2}{*}{$P$ Value } & \multirow{2}{*}{ Mean Dose-Value } & \multicolumn{2}{|c|}{$95 \% \mathrm{Cl}$ of the Difference } \\
\hline & & & & & Lower & Upper \\
\hline HI_IMRT & 13.848 & 7 & 0.0005 & 0.19905 & 0.1651 & 0.2330 \\
\hline HI 3DCRT & 11.589 & 7 & 0.0005 & 0.20755 & 0.1652 & 0.2499 \\
\hline
\end{tabular}

3D-CRT with a $P$ value of 0.001 was better for the Rt. parotid, while the $P$ value with IMRT was 0.0005 , which is better than that of the 3D-CRT; however, in Table 4 below, the values of these parameters differ.

The $P$ values for both techniques for the Lt. parotid glands were the same, but the mean dose value for IMRT (23.60 Gy) was better than that for 3D-CRT (26.54 Gy). The difference between these numbers depends on many factors, such as the location, position, and nearness of the tumor to the OARs (Table 4).

As seen in Table 5, the comparison of 3D-CRT and IMRT plans showed that the relative volume of PTV was significantly greater with the IMRT technique than with 3D-CRT (52.15 $\pm 1.61,51.09 .4 \pm 0.74 \mathrm{~Gy}$, respectively). The use of both techniques on the eight patients had PTV95\% coverage values of $>95 \%$ of the prescription dose. These results correspond with those of Abo-Madyan et al. ${ }^{23}$

\section{Discussion}

The ideal value for the conformity index is 1 , which indicates optimum conformation. In this case, radiation beams from the Gantry are conformal with the target volume within the patient's body. A conformity index greater than 1 shows a greater irradiated volume than the target volume inside the patient's body; thus, normal tissues are also included, and a hot spot may be produced. Conversely, a conformity index less than 1 indicates partial irradiation of the target volume; hence, some parts of the target volume will not be covered by radiation beams, and a cold spot may be produced.

The results of this study explicitly showed improved dose distributions for the treatment of laryngeal cancer with IMRT compared to 3D-CRT. These results are consistent with those published by Mendenhall et al. ${ }^{24}$ These improvements concern the coverage of PTVs and the sparing of the parotid glands. The effects of doses received by all patients on the parotid glands were consistent with the planning technique. The major aim for the parotid glands was to achieve a mean dose below 26 Gy (tolerance dose value) as proposed by the Radiotherapy Oncology

Table 3. Mean Value and $P$ value of Rt. Parotids in Both 3D-CRT and IMRT Techniuqes

\begin{tabular}{|c|c|c|c|c|c|c|}
\hline & \multicolumn{6}{|c|}{ Test Value $=\mathbf{0}$} \\
\hline & \multirow{2}{*}{$\mathbf{T}$} & \multirow{2}{*}{ df } & \multirow{2}{*}{$P$ Value } & \multirow{2}{*}{ Mean Dose Value Gy } & \multicolumn{2}{|c|}{$95 \% \mathrm{Cl}$ of the Difference } \\
\hline & & & & & Lower & Upper \\
\hline Rt. parotid, 3D-CRT & 5.732 & 7 & 0.001 & 2354.25000 & 1382.9722 & 3325.5278 \\
\hline Rt. parotid, IMRT & 20.423 & 7 & 0.0005 & 2411.25000 & 2132.0760 & 2690.4240 \\
\hline
\end{tabular}

Table 4. Mean Dose and $P$ Values of Lt. Parotids in Both 3D-CRT and IMRT Techniuqes

\begin{tabular}{|c|c|c|c|c|c|c|}
\hline & \multicolumn{6}{|c|}{ Test Value $=0$} \\
\hline & \multirow{2}{*}{$\mathbf{T}$} & \multirow{2}{*}{ df } & \multirow{2}{*}{$P$ Value } & \multirow{2}{*}{ Mean Dose Value Gy } & \multicolumn{2}{|c|}{$95 \% \mathrm{Cl}$ of the Difference } \\
\hline & & & & & Lower & Upper \\
\hline Rt. parotid, 3D-CRT & 9.758 & 7 & 0.0005 & 2654.62500 & 2011.3339 & 3297.9161 \\
\hline Rt. parotid, IMRT & 43.571 & 7 & 0.0005 & 2360.37500 & 2232.2763 & 2488.4737 \\
\hline
\end{tabular}


Table 5. Mean and SD (Range) for Mean Dose Gy, D2\% Gy, D98\% Gy, and D95\% Gy in Both 3DCRT and IMRT Techniques for All 8 patients

\begin{tabular}{|c|c|c|c|c|c|c|c|c|}
\hline \multirow{2}{*}{ No. } & \multicolumn{2}{|c|}{ Mean Dose PTV Gy } & \multicolumn{2}{|c|}{ D2\% Gy } & \multicolumn{2}{|c|}{ D98\% Gy } & \multicolumn{2}{|c|}{ D95\% Gy } \\
\hline & 3DCRT & IMRT & 3DCRT & IMRT & 3DCRT & IMRT & 3DCRT & IMRT \\
\hline 1 & 50.88 & 53.64 & 58.90 & 58.34 & 43.29 & 47.34 & 46.41 & 48.99 \\
\hline 2 & 51.28 & 51.72 & 54.39 & 54.71 & 45.21 & 45.86 & 47.17 & 47.99 \\
\hline 3 & 52.77 & 51.26 & 54.81 & 54.82 & 46.31 & 46.91 & 47.28 & 47.86 \\
\hline 4 & 50.58 & 49.61 & 55.34 & 54.35 & 43.73 & 43.03 & 46.78 & 45.05 \\
\hline 5 & 51.23 & 50.73 & 53.43 & 55.83 & 45.59 & 46.83 & 46.48 & 47.80 \\
\hline 6 & 50.98 & 54.20 & 54.14 & 58.15 & 45.23 & 48.95 & 46.73 & 50.21 \\
\hline 7 & 50.52 & 53.68 & 54.75 & 59.43 & 41.85 & 46.41 & 47.05 & 47.82 \\
\hline 8 & 50.49 & 52.41 & 53.48 & 54.73 & 48.71 & 49.69 & 46.99 & 50.43 \\
\hline Mean & 51.09 & 52.15 & 54.90 & 56.295 & 44.99 & 46.87 & 46.8625 & 48.26 \\
\hline SD & 0.74 & 1.61 & 1.74 & 2.02 & 2.08 & 2.01 & 0.31 & 1.68 \\
\hline
\end{tabular}

\section{Group (RTOG).}

For the eight evaluated patients, the plans showed that OAR values were below the defined dose limits when the IMRT technique was used. In 3D-CRT plans, OAR values were reasonable for parotid gland doses. Therefore, IMRT is a better option for the protection of healthy tissue in the larynx cancer region. In addition, IMRT is well known to provide better target volume coverage which is difficult in laryngeal cancer regions, because critical structures are very close to or in the target area. The current results agree with those of Emami ${ }^{25}$ and Bucci et al. ${ }^{26}$ Minimum and D95\% dose values were higher and dose homogeneity was better with the IMRT technique as shown in Table 1. Lower overall areas of hot spots and lower maximum doses with IMRT are other important results considering the side effects. For the target volumes, the main differences were seen in D max and V95\% dose values of PTV and D max, $\mathrm{D}$ min, and V95\% dose values of PTV as shown in Tables 1 and 5 .

\section{Conclusion}

Head and neck radiotherapy is one of the most challenging treatments in radiation ontology because of the number of organs with different tolerance doses, large treatment areas, complex patient anatomy, and the OARs. Based on the current results, IMRT is the best treatment planning for head and neck cancers in comparison with 3D-CRT. In this study, it was determined that intensity-modulated radiation therapy can improve the quality of life of head and neck cancer patients who have already undergone radiation therapy. These improvements concern the coverage of PTVs and saving healthy tissue, such as parotid glands.

\section{Authors' Contributions}

DKI: Study conception and design, acquisition of data, data analysis and interpretation, manuscript writing and critical revisions. FFH: acquisition of data and critical revisions of the manuscript for intellectual content. Both authors read and approved the final manuscript.

\section{Conflict of Interest Disclosures}

The authors declare that they have no conflicts of interest.

\section{Ethical Approval}

Ethical approval was obtained from the Research Ethics Committee of the College of Medicine, Hawler Medical University, Kurdistan region, Irbil, Iraq.

\section{Acknowledgments}

The authors thank all staff members of the Zhianawa

\section{Research Highlights}

\section{What Is Already Known?}

Radiotherapy is the main treatment used to preserve the larynx. There are many radiotherapy techniques, including 3D-CRT, IMRT, and VMAT. Radiation therapy for laryngeal cancer is usually given with a total dose of 50 Gy (1 Gy = $100 \mathrm{Rad}$ ) in daily fractions (200 rad per day), 5 days per week, for about 5 weeks. When radiation is used as the main treatment for cancer of the larynx, the dose of radiation may also damage the parotid glands. The best radiotherapy treatment method for all types of cancers is individualization of treatment based on a description of the characteristics of the patient, his or her disease, treatment volume, etc. Individualization depends on the individual dose distribution and individual fractionation, the homogeneity index of radiation, and the conformity index for the target region and in healthy tissue.

\section{What This Study Adds?}

This study aimed to determine which technique is suitable for treatment of the larynx, causing minimum damage to the parotid glands, investigate radiation doses on parotid glands, and compare them with the tolerance dose value of parotid Gy. The current results confirm those of several researchers who have reported that the risk to the parotid glands was significantly reduced at doses less than a few Gy with IMRT when this technique was compared with 3D-CRT. 
Cancer Center, Sulaymaniyah, Iraq, for their cooperation and facilities in carrying out this research.

\section{References}

1. Herrassi MY, Bentayeb F, Malisan MR. Comparative study of four advanced $3 \mathrm{~d}$-conformal radiation therapy treatment planning techniques for head and neck cancer. J Med Phys. 2013;38(2):98-105. doi:10.4103/0971-6203.111331.

2. Lee N, Puri DR, Blanco Al, Chao KS. Intensity-modulated radiation therapy in head and neck cancers: an update. Head Neck. 2007;29(4):387-400. doi:10.1002/hed.20332.

3. IMRT - Intensity-Modulated Radiation Therapy. https://www. radiologyinfo.org/en/info.cfm?pg=imrt. Accessed June 20, 2019.

4. Bakiu E, Telhaj E, Kozma E, Ruçi F, Malkaj P. Comparison of 3D CRT and IMRT tratment plans. Acta Inform Med. 2013;21(3):211212. doi:10.5455/aim.2013.21.211-212.

5. Cho B. Intensity-modulated radiation therapy: a review with a physics perspective. Radiat Oncol J. 2018;36(1):1-10. doi:10.3857/roj.2018.00122.

6. Grégoire V, Mackie TR. Dose prescription, reporting and recording in intensity-modulated radiation therapy: a digest of the ICRU Report 83. Imaging Med. 2011;3(3):367-373. doi:10.2217/iim.11.22.

7. Mutic S, Purdy JA, Michalski JM, Perez CA. The simulation process in the determination and definition of the treatment volume and treatment planning. In: Levitt SH, Purdy JA, eds. Technical Basis of Radiation Therapy: Practical Clinical Applications. Berlin, Heidelberg: Springer; 2006. p. 107-133. doi:10.1007/3-540-35665-7_6.

8. Morley L, Cashell A. Collaboration in health care. J Med Imaging Radiat Sci. 2017;48(2):207-216. doi:10.1016/j. jmir.2017.02.071.

9. Vaezzadeh SA, Allahverdi M, Nedaie HA, Aghili M, Esfehani M. Comparison of conventional and 3D conformal treatments using linac energies for prostate cancer. Iran J Radiat Res. 2012;10(3-4):145-150.

10. Hrinivich WT, McNutt TR, Meyer JJ. Radiation treatment planning with embedded dose escalation. Radiat Oncol. 2019;14(1):145. doi:10.1186/s13014-019-1348-3.

11. Thompson RF. RadOnc: an R package for analysis of dosevolume histogram and three-dimensional structural data. Journal of Radiation Oncology Informatics, 2014;6(1):98-110. doi:10.5166/jroi-6-1-25.

12. Fogliata A, Cozzi L, Bieri S, Bernier J. Critical appraisal of a conformal head and neck cancer irradiation avoiding electron beams and field matching. Int J Radiat Oncol Biol Phys. 1999:45(5):1331-1338. doi:10.1016/s0360-3016(99)00319-3.

13. McMenamin E. Long Term Effects of Radiation to the Head and
Neck. University of Pennsylvania - Oncolink; 2017.

14. Elekta Product. XiO Treatment Planning System TPS (version 5.00.01. Stockholm, Sweden: Elekta AB; 2014.

15. Lalya I, Marnouche EA, Abdelhak M, et al. Radiotherapy of nasopharyngeal cancer using Rapidarc: dosimetric study of military teaching hospital Mohamed V, Morocco. BMC Res Notes. 2017;10(1):112. doi:10.1186/s13104-017-2430-2.

16. Tsubouchi T, Yagi M, Sumida I, Tamari K, Seo Y, Ogawa K. The effect of beam shape on physical parameters of head and neck simultaneous-integrated boost intensity-modulated radiation therapy. Rep Pract Oncol Radiother. 2018;23(5):425-432. doi:10.1016/j.rpor.2018.08.008.

17. Frassica DA. General principles of external beam radiation therapy for skeletal metastases. Clin Orthop Relat Res. 2003(415 Suppl):S158-164. doi:10.1097/01.blo.0000093057.96273.fb.

18. Court LE, Balter P, Mohan R. Principles of IMRT. In: Nishimura $\mathrm{Y}$, Komaki R, eds. Intensity-Modulated Radiation Therapy: Clinical Evidence and Techniques. Tokyo: Springer Japan; 2015:15-42. doi:10.1007/978-4-431-55486-8_2.

19. McQuaid D, Webb S. IMRT delivery to a moving target by dynamic MLC tracking: delivery for targets moving in two dimensions in the beam's eye view. Phys Med Biol. 2006;51(19):4819-4839. doi:10.1088/0031-9155/51/19/007.

20. Knöös T, Kristensen I, Nilsson P. Volumetric and dosimetric evaluation of radiation treatment plans: radiation conformity index. Int J Radiat Oncol Biol Phys. 1998;42(5):1169-1176. doi:10.1016/s0360-3016(98)00239-9.

21. Yoon $M$, Park SY, Shin D, et al. A new homogeneity index based on statistical analysis of the dose-volume histogram. J Appl Clin Med Phys. 2007;8(2):9-17. doi:10.1120/jacmp.v8i2.2390.

22. Petrova D, Smickovska S, Lazarevska E. Conformity index and homogeneity index of the postoperative whole breast radiotherapy. Open Access Maced J Med Sci. 2017;5(6):736739. doi:10.3889/oamjms.2017.161.

23. Abo-Madyan Y, Polednik M, Rahn A, Schneider F, Dobler B, Wenz F, Lohr F. Improving dose homogeneity in large breasts by IMRT: efficacy and dosimetric accuracy of different techniques. Strahlenther Onkol. 2008;184(2):86-92. doi:10.1007/s00066008-1730-9.

24. Mendenhall WM, Amdur RJ, Palta JR. Intensity-modulated radiotherapy in the standard management of head and neck cancer: promises and pitfalls. J Clin Oncol. 2006;24(17):26182623. doi:10.1200/jco.2005.04.7225

25. Emami B. Tolerance of normal tissue to therapeutic radiation. Reports of Radiotherapy and Oncology. 2013;1(1):35-48.

26. Bucci MK, Bevan A, Roach M 3rd. Advances in radiation therapy: conventional to $3 \mathrm{D}$, to IMRT, to $4 \mathrm{D}$, and beyond. CA Cancer J Clin. 2005;55(2):117-134. doi:10.3322/ canjclin.55.2.117. 